\title{
Sidelobe Reduction by Subarray Stacking for Uniformly Excited mmW Phased Arrays
}

\author{
Muhammad Yasir Javed, Nuutti Tervo, Marko E. Leinonen and Aarno Pärssinen \\ Centre for Wireless Communications (CWC), University of Oulu, Finland \\ \{yasir.javed, nuutti.tervo, marko.e.leinonen, aarno.parssinen\}@oulu.fi
}

\begin{abstract}
The performance of multi-user millimeterwave $(\mathrm{mmW})$ systems is limited by relatively high sidelobe levels (SLLs) of antenna arrays. Per-antenna amplitude control can be used to adjust the amplitudes to reduce the SLL, but the reduction is often achieved at the cost of reduced transmitted power. Large two-dimensional (2D) antenna panels used in $\mathrm{mmW}$ phased arrays, however, allow the $2 \mathrm{D}$ antenna configuration to be reconfigured to reduce the SLL. In this paper, we present a simplified approach for sidelobe reduction by stacking multiple uniform linear arrays of different size to reduce the sidelobes across the horizontal plane. The approach is based on the relation between the number of antenna elements and the directions of null and sidelobe maxima. The sidelobe reduction is demonstrated by both simulations and measurements. The measurements are carried out in an anechoic chamber at $28 \mathrm{GHz}$ center frequency using a $100 \mathrm{MHz}$ wide modulated 5GNR waveform.
\end{abstract}

Index Terms-Amplitude tapering, array thinning, twodimensional array, space tapering, 5G, phased array.

\section{INTRODUCTION}

Adoption of millimeter-wave $(\mathrm{mmW})$ is one of the key fifth generation (5G) communication enablers due to its potential to achieve higher throughput required by the future networks [1], [2]. The increase in throughput is achieved by large available bandwidth together with highly directional antenna arrays used to manage the interference and to increase the signal-to-interference-plusnoise-ratio (SINR). Moreover, the $\mathrm{mmW}$ antenna size and inter-element spacing are in the order of millimeters which makes it possible to pack hundreds of elements in compact form factor.

In radio frequency (RF) beamforming, unitary amplitude excitation over the antenna elements is usually preferred because of per-antenna power constraint dictated by the power amplifier (PA) in the transmitter and noise figure (NF) in the receiver. Also, unitary amplitude excitation is known to give the most narrow beamwidth with the expense of a fixed relatively high sidelobe level (SLL) (theoretically $13.3 \mathrm{~dB}$ below the main lobe). In multibeam case where various beams carry different data streams, the sidelobes interfere with the main lobes of the other beams, causing inter-beam interference (IBI). IBI restricts the SINR, which results in limited datarate per beam. To maximize the data rate of each user, SINR must also be maximized. Therefore, $\mathrm{mmW}$ cellular systems require narrow beams with low sidelobe level to maximize the usage of the available antenna gain in a certain direction while causing minimum interference in other directions.

Various techniques, e.g. inter-beam interference cancellation [3] or alike zero-forcing precoding [4], can be used to reduce the interference in known directions. However, in practice, the performance of such techniques depends highly on the quality of the channel state information which is never perfect in practical systems. Another approach for interference reduction is to reduce the maximum interference which is often dominated by the SLL. Most of the existing sidelobe reduction techniques were originally developed for radar and satellite communication and are not designed for compact cellular systems. These techniques are now expected to be used in cellular systems due to the necessity of phased arrays.

In general, sidelobe reduction techniques can be divided into two main categories: (i) varying amplitude excitation over the antenna elements in an array (amplitude tapering [5]) and (ii) using nonidentical spacing between elements, e.g. array thinning [6]. In amplitude tapering, the excitation amplitudes are varied from element to element by using window functions such as binomial, Chebyshev or Taylor. The cost of changing amplitude distribution to be different from unitary is the higher beamwidth. Furthermore, especially in the transmitter (Tx) side, the gain control will often result in smaller maximum effective isotropic radiated power (EIRP) due to the maximum available power per single branch. [3]

Another approach to reduce the SLL is to use nonidentical spacing between the elements [6]. However, if the inter-element spacing is increased from the traditional half of a wavelength, the beamwidth decreases [7] and grating lobes occur. On the other hand, decreasing the inter-element spacing from the half of a wavelength increases the coupling between antenna elements [8]. The finite antenna coupling causes challenges due to the circulator-free array architectures used in highly integrated $\mathrm{mmW}$ systems [9]. Large planar arrays can also be partitioned into two-dimensional aperiodic tiles to improve the peak sidelobe levels, directivity and aperture efficiency [10], [11]. However, such techniques are only alternative ways of achieving array thinning (i.e. mean inter-element spacing is more than half a wavelength). 


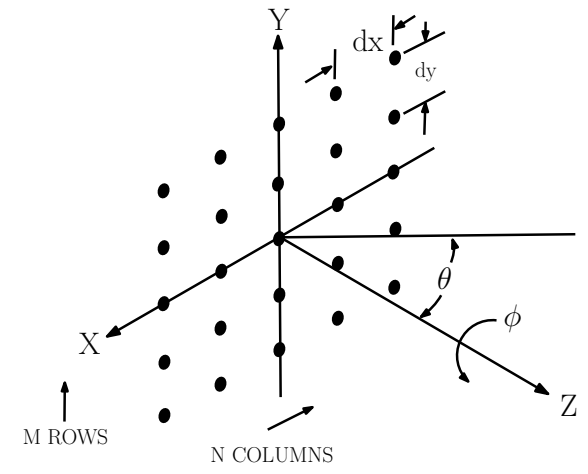

Fig. 1. Two-dimensional antenna panel with vertically stacked ULAs with different number of elements.

With aperiodic geometries it is challenging to avoid the grating lobes when the beam is electronically steered and the scanning range is often limited.

In this paper, we propose a technique to reconfigure the antenna elements in two-dimensional array to reduce the SLLs across the azimuth plane. The technique can be used to configure large antenna panels with uniform inter-element spacing to reduce the interference without leaving dummy elements in the center of the array as e.g. in [12]. The rest of the paper is organized as follows. Section II presents the basic idea and the flow of the proposed sidelobe reduction technique. Section III presents the experimental validation of the proposed technique. The conclusions are drawn in Section IV.

\section{Sidelobe Reduction by Null Placement FROM MULTIPLE STACKED ULAS}

\section{A. Direction of Nulls and Sidelobes of Antenna Array}

Consider a planar array with $\mathrm{M}$ rows and $\mathrm{N}$ columns of antenna elements arranged along a rectangular grid with equal inter-element spacing along both axis $\left(d_{x}=d_{y}=\right.$ $\lambda / 2$ ), where $\lambda$ is the wavelength at center frequency) shown in Fig. 1. The rows of the array located in the x-axis are $N_{m}$-element uniform linear arrays (ULAs) stacked along the y-axis. Amplitude excitation of each element is assumed to be identical in order to achieve narrow beam. Location of nulls of $m$ th uniform linear subarray in azimuth plane can be calculated as [7]

$$
\phi_{\text {nulls }}=\sin ^{-1}\left[\frac{\lambda}{2 \pi d_{x}}\left(-\beta_{\phi} \pm \frac{2 n \pi}{N_{M}}\right)\right],
$$

where $n=1,2,3, \ldots, n \neq 1 N_{M}, 2 N_{M}, 3 N_{M}, \ldots$ is the null index, and $N_{M}$ is the number of elements in $m$ th subarray and $\beta_{\phi}$ is phase progression over the ULA used for azimuth beamsteering. Similarly the location of the subarray sidelobe maxima can be calculated as

$$
\phi_{s l}=\sin ^{-1}\left[\frac{\lambda}{2 \pi d_{x}}\left(-\beta_{\phi} \pm 2 s \pi\right)\right],
$$

where $s=1,2,3, \ldots, s \neq 0$ is the sidelobe index.

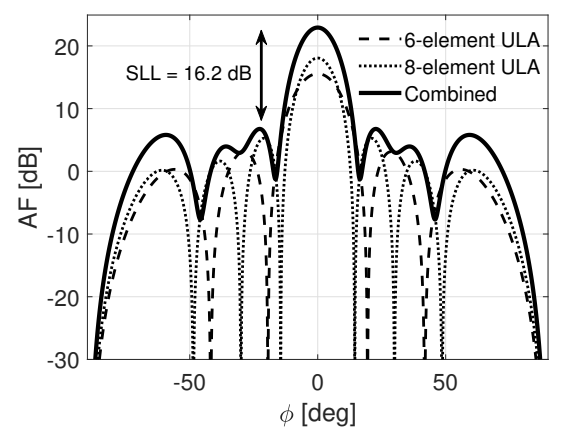

Fig. 2. Simulated array factors of six- and eight-element ULAs and the combined array pattern.

\section{B. Subarray Stacking for Sidelobe Reduction}

The sidelobes of the overall beam over the azimuth plane can be reduced by stacking multiple variously sized subarrays on top of each other. The number of elements for each row can be calculated by using equations (1) and (2). The general idea is that the number of antennas in the following subarray is increased and selected such that it places a null in the location of the first sidelobe of the previous subarray. By doing that, the main lobe power increases while the SLL remains constant. An example with two antenna rows is simulated in Fig. 2. First subarray has six elements and the next subarray hence requires eight elements to place a null in the direction of the first sidelobe of the first subarray. The location of the sidelobes of each subarray is different. When the subarrays of different size are combined, the sidelobe power increases less than the main lobe power, resulting in reduced SLL. The flow diagram of the subarray stacking is presented in Fig. 3. The total number of antenna elements $\left(N_{R E Q}\right)$ can be defined for example to achieve the requested link range.

\section{Relaxation of the Null Direction}

If the sidelobe level is not allowed to be increased, the equation (2) may result in unfeasible number of elements. However, the widths of the nulls and sidelobes depend on the number of elements. The target null direction is therefore not required to be exactly in the direction of the other sidelobe maxima and the tolerable error depends on the widths of the nulls and maxima. To take this into account and avoid excessive number of antenna elements that is a consequence of too precise null positioning, the target direction of the null for the following subarray can be relaxed such that $\phi_{\text {null }} \in \phi_{\text {margin }}$. The idea of the null margin $\phi_{\text {margin }}$ is demonstrated in Fig. 4. ULAs have $N_{M}-2$ sidelobes having width of approximately $w_{s l}=2 \pi / N_{M}$ [7]. In this paper, we use a threshold $\gamma$ to describe the relaxation of 


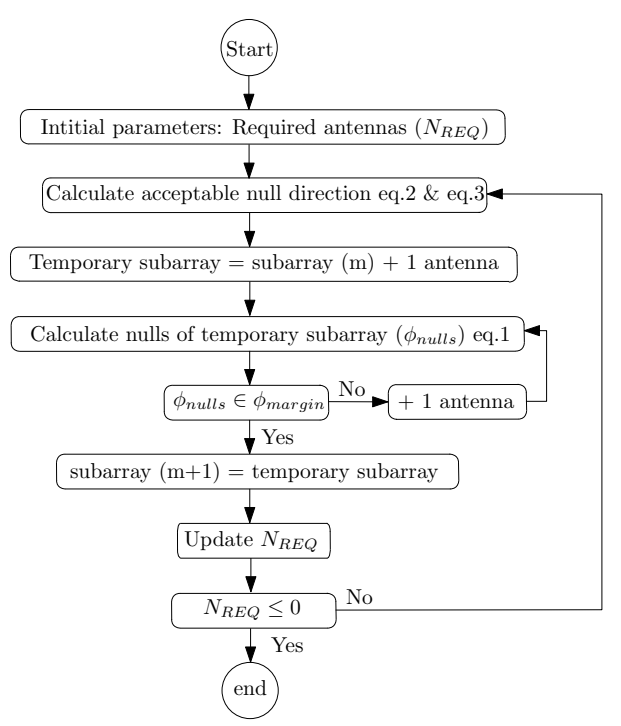

Fig. 3. Flow diagram of the subarray stacking procedure for sidelobe reduction.

the target null direction. The acceptable null direction for the $m+1$ antenna row is therefore defined as

$$
\phi_{\text {margin }}(m+1)=\phi_{s l}(m)+\left[-\gamma w_{s l}(m), \gamma w_{s l}(m)\right]
$$

where $w_{s l}$ is the width of first sidelobe of $m$ th array. Equation (3) calculates the null margin for subarray $m+1$ from the maximum sidelobe direction of subarray $m$. Hence, for example $\gamma=0.1$ denotes that the null direction must fall into $\pm 10 \%$ of the sidelobe's $3 \mathrm{~dB}$ beamwidth from the sidelobe maxima. Fig. 5 presents simulations results of the total number of antenna elements of the 2D array as a function of the SLL with different $\gamma$ values. Naturally, the wider null margin leads to smaller number of antenna elements from subarray to subarray and vice versa, hence reducing the total number of elements. However, increasing the margin $\gamma$ also decreases the sidelobe reduction performance with higher SLL targets. Hence, $\gamma$ can be used to compromise between the array size and SLL reduction performance. Simulation results also show that if more subarrays are stacked, SLL can be further reduced. The potential of the proposed technique is also shown in Fig. 5 as it gives $30 \mathrm{~dB}$ of SLL for eight vertically stacked subarrays with 100 antenna elements.

\section{Demonstration and Measurements}

In order to demonstrate the sidelobe reduction technique in practice, we use a 64-element antenna array [13] designed originally for mobile backhaul applications. The array is divided into $2 \times 2$ groups denoted as unit cells shown in Fig. 6a. Each unit cell has four linearly polarized patch antenna elements slanted to $-45^{\circ}$ with respect to the vertical. The spacing between the elements is half a wavelength $(=5.4 \mathrm{~mm})$ at $28 \mathrm{GHz}$. The simulated radiation pattern of a single unit cell is shown in

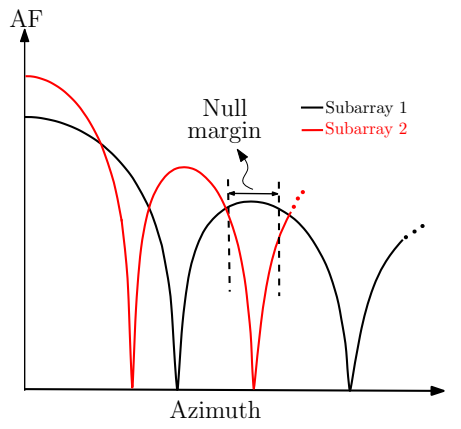

Fig. 4. Margin for relaxing the null direction.

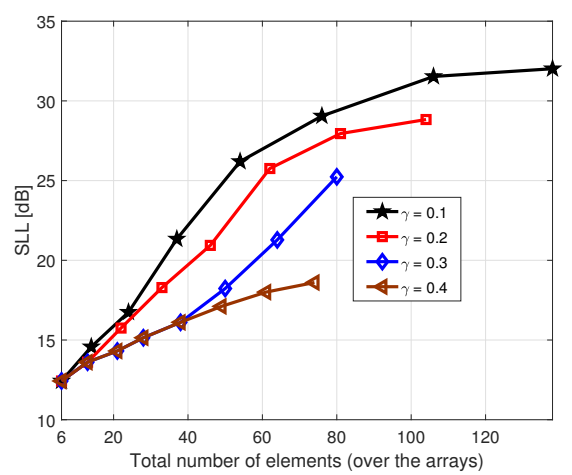

Fig. 5. Size of subarrays and relative SLL with different relaxation $(\gamma)$ for null directions, simulations are made upto 8 rows of antennas

Fig. 6b. 16 unit cells are placed to $8 \times 2$ array formation with spacing of $\lambda$ between the cells. Hence, in total the array has 64 individual elements with 16 transceiver (TRx) ports. The array is driven with 16-chain phased array TRx [14], [15]. The simplified block diagram of the transceiver is shown in Fig. 7. The TRx switches of each antenna branch are used to switch branches on and off in order to configure the 2D shape of the antenna array. In the measurements, the transceiver array is operating in the receive mode.

The block diagram of the measurement setup located in an anechoic chamber is presented in Fig. 9. Each of the 16 TRx banches has individual TRx switches, low noise amplifiers (LNAs), PAs and 5-bit phase shifters. Keysight M8190A arbitrary waveform generator (ARB) an E8257B PSG signal generator are used to generate a $100 \mathrm{MHz}$ wide 16-quadrature amplitude modulation (QAM) cyclic-prefix orthogonal frequency division multiplexing (CP-OFDM) waveform at $28 \mathrm{GHz}$ center frequency following the $3 \mathrm{GPP} / \mathrm{NR}$ standard. In the transmission, the signal is amplified by CA2630-141 external amplifier to a A-info LB-28-15 horn antenna located at 2 meter distance in the far-field of the phased array receiver (DUT). The phased array receiver output is fed to UXA signal analyzer which is used to measure channel power of the signal over the $100 \mathrm{MHz}$ band. The 


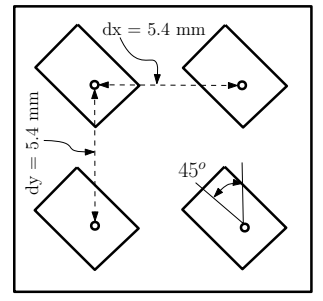

(a)

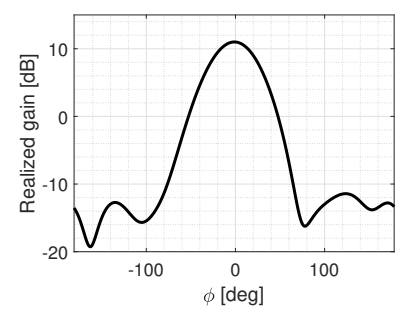

(b)
Fig. 6. (a) $2 \times 2$ unit cell antenna array with single feed and (b) horizontal cut of the simulated radiation pattern of the unit cell.

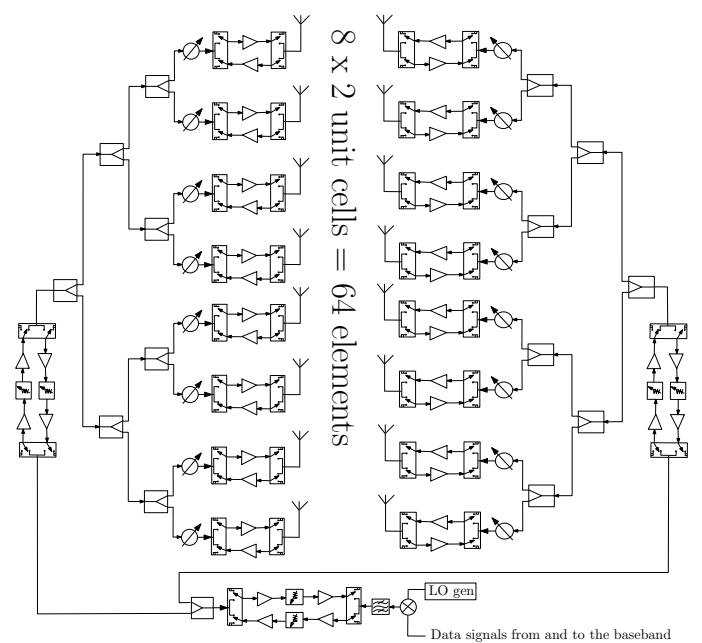

Fig. 7. Simplified block diagram phased array transceiver under test.

DUT is placed on a rotating table in order to measure the radiation pattern over the azimuth quarter plane. The measurements are conducted at $28 \mathrm{GHz}$ center frequency with 1 degree angle resolution over $\phi \in\left[-45^{\circ}, 45^{\circ}\right]$. For simplicity, the phase shifters are calibrated to the 0 degree azimuth angle. However, it should be noted that the measured sidelobe configuration works also when the beam is steered in azimuth domain. A photograph of the measurement setup in the chamber is shown in Fig. 8.

The simulations are conducted in MATLAB by using the radiation pattern of the unit cell simulated in Computer Simulation Technology (CST) microwave studio and the well-known array factor principle. The simulations are performed with a continuous-wave $(\mathrm{CW})$ signal while the measurements are conducted with modulated signal. Due to the limitation of only 16 ports in the transceiver array (with two independent rows), only one configuration is demonstrated. The chosen configuration based on the method presented in this paper is depicted in Fig. 10a. The demonstrated configuration has four $2 \times 2$ antenna unit cells in the first subarray and six in the second one. In total, 40 antenna elements are active. For the comparison, we also measured a rectangular configuration with 40 elements in $10 \times 4$ formation.

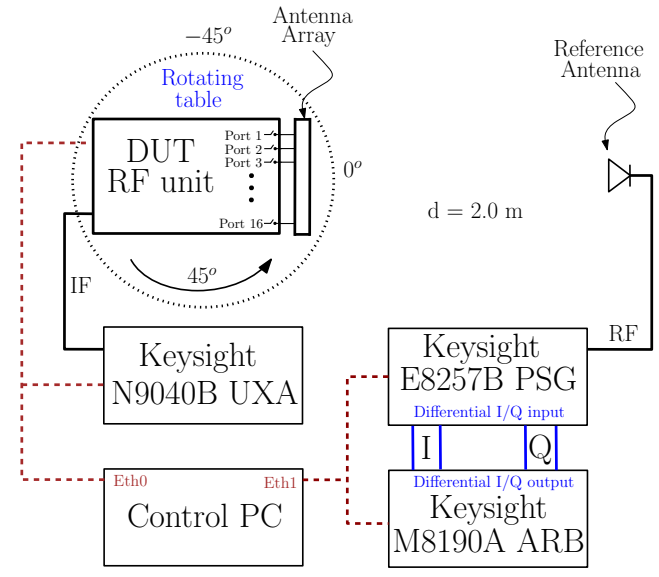

Fig. 8. Block diagram of the OTA-measurement setup with $100 \mathrm{MHz}$ wide $5 \mathrm{GNR}$ waveform at $28 \mathrm{GHz}$.

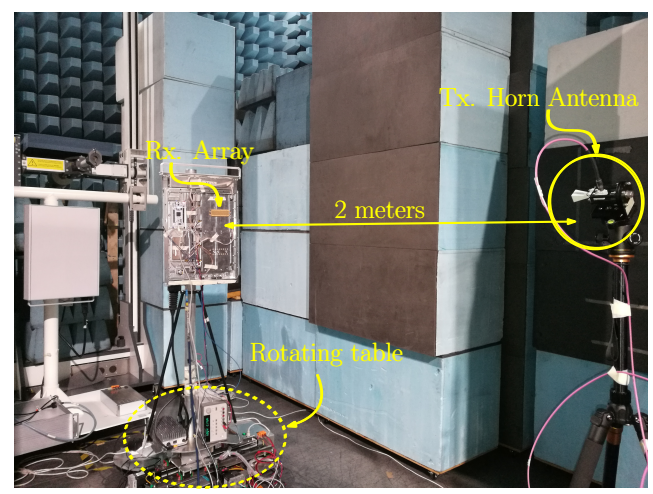

Fig. 9. Photograph of the measurement arrangement in the anechoic chamber.

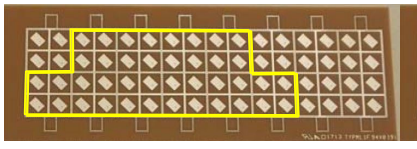

(a)

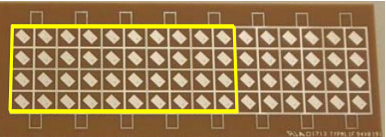

(b)
Fig. 10. $28 \mathrm{GHz}, 64$-element, 16-chain phased array with (a) the proposed configuration and (b) rectangular 10x4, respectively.

The simulation and measurement results are presented in Fig. 11. The radiation patterns of the both measured configurations are not symmetric due to the antenna implementation and non-equal gains of the receiver branches [14], [15]. In the measurements, the receiver gain and the gain of the horn antenna is compensated from the results. The notches are not exactly at the same positions in the measurements and simulations. However, the first sidelobe of the first array falls approximately to the direction of the first null of the second subarray and hence the sidelobes are reduced with the proposed configuration. The SLL results of maximum right and left sidelobe levels are collected to the Table I. The simulations show that the proposed configuration has 


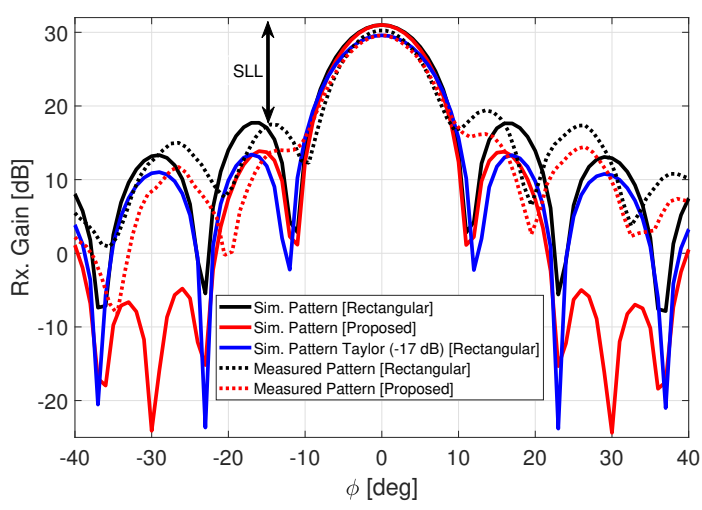

Fig. 11. Horizontal cuts of the simulated and the measured radiation patterns of the rectangular and the proposed antenna configuration.

TABLE I

Simulated AND MEASURED SLLS OF PROPOSED (PROP) AND RECTANGULAR (RECT) ANTENNA CONFIGURATIONS

\begin{tabular}{|c|c|c|c|}
\hline Sim. Rect (right) & $13.4 \mathrm{~dB}$ & Meas. Rect (right) & $10.9 \mathrm{~dB}$ \\
\hline Sim. Rect (left) & $13.3 \mathrm{~dB}$ & Meas. Rect (left) & $12.8 \mathrm{~dB}$ \\
\hline Sim. Prop (right) & $17.2 \mathrm{~dB}$ & Meas. Prop (right) & $13.4 \mathrm{~dB}$ \\
\hline Sim. Prop (left) & $17.1 \mathrm{~dB}$ & Meas. Prop (left) & $15.4 \mathrm{~dB}$ \\
\hline
\end{tabular}

potential for reducing the first sidelobe level by around $4 \mathrm{~dB}$. The measurements show 2.5 and $2.6 \mathrm{~dB}$ sidelobe reduction with only two rows of unit cells. The loss in the reduction performance is partly caused by the wideband measurement with modulated signal which decreases the notch depth when the power is integrated over the whole $100 \mathrm{MHz}$ signal band. Amplitude tapered pattern with Taylor window ( $-17 \mathrm{~dB}$ SLL target) is also depicted in the Fig. 11. Compared to the proposed antenna selection method, amplitude tapering decreases SLL at the expense of wider beam width and reduced directivity. Both simulation and measurement results indicate that the proposed sidelobe reduction technique works in practice. With larger number of rows, low sidelobe levels can be achieved to reduce the interference even further.

\section{CONCLUSION}

We propose a sidelobe reduction technique, which stacks multiple ULAs in the vertical domain in order to reduce the sidelobes over the azimuth plane. In phased arrays, where rectangular antenna panel is shared with multiple beams, can be implemented in any frequency range. The number of antennas in each row is varied in order to align the nulls and maxima of the rows such that the sidelobe power is not increased while the main lobe power raises as a function of antenna elements. Threshold parameter for the acceptable region of null direction is selected based on the widths of the nulls and sidelobes. The sidelobe reduction is demonstrated by using a 64element phased array transceiver operating at $28 \mathrm{GHz}$ center frequency. Over-the-air measurements are carried out in an anechoic chamber by using $100 \mathrm{MHz}$ wide CPOFDM waveform following the 3GPP/NR standard. The configuration proposed by the subarray stacking method is compared against rectangular array with the same total number of antenna elements. Compared to the reference rectangular antenna configuration, the measurement results show $2.5 \mathrm{~dB}$ of sidelobe reduction with two rows of antennas while the simulations reveals even $4 \mathrm{~dB}$ of sidelobe reduction potential with only two rows. The simulations also show that SLL can be further reduced if more subarrays are stacked. For example, with 128 antennas $\sim 30 \mathrm{dBc}$ sidelobe level is achievable.

\section{ACKNOWLEDGMENT}

This research has been financially supported by Nokia Corporation Ltd, Academy of Finland 6Genesis Flagship (grant 318927) and Business Finland project 5GForce.

\section{REFERENCES}

[1] Z. Pi and F. Khan, "An Introduction to Millimeter-wave Mobile Broadband Systems," in IEEE Communications Magazine, vol. 49, no. 6, pp. 101-107, June 2011.

[2] S. Rangan, T. S. Rappaport and E. Erkip, "Millimeter-Wave Cellular Wireless Networks: Potentials and Challenges," in Proceedings of the IEEE, vol. 102, no. 3, pp. 366-385, March 2014.

[3] Javed, M., Tervo, N., Pärssinen, A. (2018). Inter-beam Interference Reduction in Hybrid mmW Beamforming Transceivers.

[4] A. Wiesel, Y. C. Eldar and S. Shamai, "Zero-Forcing Precoding and Generalized Inverses," in IEEE Transactions on Signal Processing, vol. 56, no. 9, pp. 4409-4418, Sept. 2008.

[5] C. L. Dolph, "A Current Distribution for Broadside Arrays Which Optimizes the Relationship between Beam Width and Side-Lobe Level," in Proceedings of the IRE, vol. 34, no. 6, pp. 335-348, June 1946.

[6] A. Ishimaru, "Theory of Unequally-spaced Arrays," in IRE Transactions on Antennas and Propagation, vol. 10, no. 6, pp. 691-702, November 1962.

[7] Balanis, C. (2012). Antenna Theory: Analysis and Design. 3rd ed. New York: Wiley.

[8] S. Edelberg and A. Oliner, "Mutual Coupling Effects in Large Antenna Arrays: Part 1-Slot arrays," in IRE Transactions on Antennas and Propagation, vol. 8, no. 3, pp. 286-297, May 1960.

[9] A. Prata et al., "Towards Circulator-free Multi Antenna Transmitters for 5G," 2017 IEEE MTT-S International Microwave Symposium (IMS), Honololu, HI, 2017, pp. 677-680.

[10] T. Isernia, M. D'Urso and O. M. Bucci, "A Simple Idea for an Effective Sub-Arraying of Large Planar Sources," in IEEE Antennas and Wireless Propagation Letters, vol. 8, pp. 169-172, 2009.

[11] V. Pierro et al., "Radiation Properties of Planar Antenna Arrays based on Certain Categories of Aperiodic Tilings," in IEEE Transactions on Antennas and Propagation, vol. 53, no. 2, pp. 635-644, Feb. 2005.

[12] S. E. Nai, W. Ser, Z. L. Yu and H. Chen, "Beampattern Synthesis for Linear and Planar Arrays With Antenna Selection by Convex Optimization," in IEEE Transactions on Antennas and Propagation, vol. 58, no. 12, pp. 3923-3930, Dec. 2010.

[13] M. Sonkki et al., "Linearly Polarized 64-element Antenna Array for mm-wave Mobile Backhaul Application, "12th European Conference on Antennas and Propagation (EuCAP 2018), London, 2018, pp. 1-5.

[14] M. E. Leinonen et al., $28 \mathrm{GHz}$ Wireless Backhaul Transceiver Characterization and Radio Link Budget, ETRI Journal, vol. 40, no. 15, pp. 89100, Feb. 2018.

[15] O. Kursu et al., Design and Measurement of a $5 \mathrm{G} \mathrm{mmW} \mathrm{Mobile}$ Backhaul Transceiver at $28 \mathrm{GHz}$, EURASIP Journal on Wireless Communications and Networking, vol. 2018, no. 1, p. 201, Aug 2018 . 